\title{
Identifikation von Fahrertypen im Kontext des automatisierten Fahrens
}

\section{Ergebnisse einer Online-Befragung und einer Fahrsimulator-Studie}

\author{
Jan Schlüter ${ }^{1}$ (D) - Marco Hellmann ${ }^{1}$ · Johannes Weyer ${ }^{1}$ \\ Eingegangen: 14. Juli 2020 / Angenommen: 9. Juni 2021 / Online publiziert: 6. Juli 2021 \\ (c) Der/die Autor(en) 2021
}

\section{Zusammenfassung}

Im Straßenverkehr existieren unterschiedliche Konzepte zur Identifikation von Fahrertypen, die sich hinsichtlich Fahrverhalten und Einstellung zum Fahren unterscheiden. Im Rahmen der Automatisierung von Fahraufgaben gilt es zu überprüfen, wie diese Konzepte an die Herausforderungen veränderter Mensch-Maschine-Interaktion angepasst werden müssen und ob sich neuartige Fahrertypen identifizieren lassen. Auf Basis bestehender Typisierungen aus der Verkehrspsychologie sowie Erkenntnissen der Automationsforschung werden dazu die Konzepte des „Driving Style“ und „Driving Skill“ weiterentwickelt, um Fahrertypen im Kontext des automatisierten Fahrens zu identifizieren. In einer großzahligen Online-Umfrage wurden drei Fahrertypen identifiziert, die sich insbesondere hinsichtlich ihrer Einstellung zum automatisierten Fahren unterscheiden. In einer experimentellen Studie im Fahrsimulator kann anschließend gezeigt werden, dass diese Fahrertypen die Automation im Fahrzeug jeweils anders erleben und daher differenzierte Ansprüche an diese richten. Insgesamt deuten die Studienergebnisse darauf hin, dass die Akzeptanz des automatisierten Fahrens durch nutzergerechte Technik gefördert werden könnte. Die Ergebnisse dienen dazu, die jeweiligen Fahrertypen, ihre Einstellungen und ihre Nutzungspräferenzen im Kontext des automatisierten Fahrens besser zu verstehen und erste Ansatzpunkte für deren Berücksichtigung in der adaptiven Technikentwicklung zu identifizieren.

Availability of data and material: The data is confidential and not publicly available.

Die Studiendaten sind vertraulich und nicht öffentlich zugänglich.

Code availability: No custom software code has been used for research.

Für diese Studie wurde kein spezifischer Software-Code genutzt.

Jan Schlüter

jan.schlueter@tu-dortmund.de
Marco Hellmann

marco.hellmann@tu-dortmund.de

Johannes Weyer

johannes.weyer@tu-dortmund.de

1 Technology Studies Group, Faculty of Social Sciences, TU Dortmund University, 44221 Dortmund, Deutschland 


\title{
Identification of driver types in automated driving
}

Results of an online survey and a driving simulator study

\begin{abstract}
In road traffic there are different concepts for identifying driver types and classifications, which differ in driving behavior and attitude towards driving. As vehicle automation proliferates, it is important to check how these concepts have to be adapted to the challenges of changing human-machine-interaction and whether new classifications of drivers can be identified. Based on existing classifications and findings from traffic psychology and automation research, the concepts of "Driving Style" and "Driving Skill" are refined to identify different driving types in vehicle automation contexts. Based on an online survey, three driver types are identified, which differ particularly in regard to their attitude towards automated driving. In a follow-up experimental study in a driving simulator, it is shown that these driver types experience automation in a simulator vehicle differently and therefore make different claims on driving automation. Overall, the study results indicate that the acceptance of automated driving could be promoted by adaptive technologies. Results contribute to understanding different driver types, their attitudes and driving preferences when using automated vehicles and therefore may be a first approach for consideration in developing adaptive automation technologies.
\end{abstract}

\section{Einleitung}

Die zunehmende Automatisierung im Fahrzeug stellt einen zentralen Gegenstand aktueller Debatten der Verkehrsforschung dar. Die Society of Automotive Engineers (SAE) unterscheidet dazu sechs Stufen der Automatisierung von Fahraufgaben. Diese reichen von der rein manuellen Fahrzeugführung (Stufe 0) bis hin zur Übernahme aller Fahraufgaben durch das Fahrzeug (Stufe 5) [31]. Aktuell befindet sich die Entwicklung der Fahrzeugtechnik zwischen einer Teilautomatisierung und einer bedingten Vollautomatisierung. Der Übergang zum vollautonomen Fahren wird hingegen noch längere Zeit in Anspruch nehmen [30]. Damit stellt der Mensch auch mittelfristig noch eine kritische Komponente beim automatisierten Fahren dar. Dabei wird angenommen, dass sich durch die zunehmende Automatisierung im Fahrzeug bestehende Muster der Mensch-Technik-Interaktion verändern [2]. In Zukunft gilt es, die neue Definition von Rollen, Kompetenzen und Zuständigkeiten in einem solchen soziotechnischen System und der damit einhergehenden „hybriden Handlungsträgerschaft“ [28] zu berücksichtigen.

Studien aus der Verkehrspsychologie deuten dabei auf die Existenz unterschiedlicher Fahrertypen hin, die einen signifikanten Einfluss auf das Fahrverhalten und die Sicherheit im Straßenverkehr haben [7, 10, 35]. Im Rahmen der zunehmenden Automatisierung und dem sich dadurch verändernden Verhältnis zwischen Mensch und Technik im Fahrzeug ergibt sich folgende Forschungsfrage: Wie müssen bestehende Konzepte zur Fahrertypisierung an den Kontext des automatisierten Fahrens angepasst werden [3]?

Ziel des vorliegenden Beitrags ist eine erste Anpassung der bestehenden Fahrertypisierungen im Kontext des automatisierten Fahrens heuristisch vorzuschlagen und darauf aufbauend diese empirisch zu untersuchen. Dazu wird ein Fragebogen entwickelt, der auf etablier- ten Messinstrumenten zur Fahrertypisierung basiert und um ausgewählte Variablen aus der Automationsforschung ergänzt wird (Abschn. 2). Aus den Daten einer repräsentativen Online-Befragung werden mittels Clusteranalyse drei Fahrertypen identifiziert, die Rückschlüsse auf die Akzeptanz- und Einstellungsunterschiede gegenüber dem automatisierten Fahren ermöglichen (Abschn. 3). In einer experimentellen Studie wird anschließend gezeigt, dass sich diese Unterschiede auch nach einem Fahrerlebnis mit hochautomatisierten Fahrassistenzsystemen wiederfinden (Abschn. 4). Der Beitrag schließt mit Fazit und Studienlimitationen ab (Abschn. 5). Die Ergebnisse dienen dazu, die jeweiligen Fahrertypen, ihre Einstellungen und ihre Nutzungspräferenzen im Kontext des automatisierten Fahrens besser zu verstehen und erste Ansatzpunkte für deren Berücksichtigung in der adaptiven Technikentwicklung zu identifizieren.

\section{Fahrertypen im Kontext des automatisierten Fahrens}

In zahlreichen wissenschaftlichen Studien wurden Fahrertypen anhand unterschiedlicher situationsunabhängiger Merkmale bestimmt. So wurden etwa aggressives, risikoreiches, vorsichtiges und ängstliches Fahrverhalten unterschieden [34]. Als etablierte zu unterscheidende Kategorien gelten dabei „Driving Skill“ und „Driving Style“ [5, 11, 38]. In der Kategorie „Driving Style“ werden Einflussfaktoren zusammengefasst, wie der Mensch routinemäßig und gewöhnlich sein Fahrzeug führt. Diese Kategorie umfasst Variablen wie Selbsteinschätzung der eigenen Fahrweise, Risikobereitschaft oder Sensation Seeking, also die Tendenz zum abenteuerlustigen Fahren [29, 34]. Er basiert auf kognitiven, emotionalen, sensorischen und motorischen Einflussfaktoren [38]. Der Driving Style wird maßgeblich 
von der persönlichen Einstellung zum Fahren beeinflusst und wird in der deutschen Übersetzung auch als Fahrstil bezeichnet [32]. In die Kategorie „Driving Skill“ fallen hingegen von der Persönlichkeit unabhängige oder nur mittelbar abhängige Einflussfaktoren, wie gut ein Mensch sein Fahrzeug im Straßenverkehr beherrscht, beispielsweise Fahrerfahrung, Situationsbewusstsein, allgemeines Fehlerverhalten und Fehleranfälligkeit oder die Sicherheitskompetenz [23]. Der „Driving Skill“ bezeichnet also die generellen Fahrfähigkeiten und wird in der deutschen Übersetzung auch als Fahrkompetenz bezeichnet [32]. Während der „Driving Style“ eher schwierig veränderbar ist, kann sich der „Driving Skill“ durch wachsende Fahrerfahrung und Trainings verbessern [19]. In wissenschaftlichen Studien wurde nachgewiesen, dass „Driving Skill“ und „Driving Style" miteinander korrelieren [5, 11, 21, 38]. So zeigte sich, dass ein aggressiverer Fahrstil mit variableren Fahrkompetenzen einhergeht [38], während mehr Fahrerfahrung und Trainings mit einem vorsichtigeren, sichereren Fahrstil einhergehen [11]. Menschen mit geringen Fahrkompetenzen tendieren dagegen zu einem eher risikoreichen Fahrstil [23].

Vorgenannte Studien nehmen diese Kategorisierungen von Fahrertypen und Bestimmungen von Fahrverhalten vor allem im Kontext des nicht automatisierten Fahrens vor. Es existieren zudem erste Studien, die die klassischen Typisierungen auf experimentelle Simulator-Studien mit hochautomatisierten Fahrzeugen anwenden. Dabei konnten festgestellt werden, dass sich unterschiedliche Verhaltensweisen und Vertrauen der menschlichen Fahrer auch im Umgang mit automatisierten Fahrzeugen zeigen [3, 14, 33]. So zeigten Menschen mit eher komfortablem Fahrstil geringere Geschwindigkeit, weniger Beschleunigung und einen gröBeren Abstand zum nächsten Fahrzeug als Menschen mit eher dynamischem Fahrstil [3]. Das Vertrauen in das automatisierte Fahrzeug stieg, wenn der automatisierte Fahrstil hinsichtlich Geschwindigkeit, Beschleunigungsverhalten sowie Querführung an das Fahrverhalten des menschlichen Fahrers angepasst und damit personalisiert wurde [33].

Auch zeigte sich bei Fahrern mit unterschiedlichen „Driving Styles“ auch eine unterschiedliche Wahrnehmung sowie Akzeptanz bezüglich der Nutzung automatisierter Fahrzeuge: So zeigte sich, dass vorsichtigere Fahrer einen automatisierten Fahrstil bevorzugen, der ihrem eigenen ähnlich ist und entsprechend abweichende automatisierte Längsführung des Fahrzeugs wie schnelles Beschleunigen oder Abbremsen zu einer geringeren Akzeptanz des Fahrzeugs führen [12]. Weiterhin zeigte sich, dass jüngere Fahrer einen automatisierten Fahrstil bevorzugen, der ihrem eigenen ähnelt, während ältere Fahrer einen riskanteren automatisierten Fahrstil vorziehen [14]. Fahrer mit einem vorsichtigen „Driving Style“ bevorzugen in hochautomatisierten Fahr- zeugen zudem Informationsdarstellungen über Geschwindigkeit oder über menschliches Fehlverhalten - zum Beispiel zu langsames Fahren. Sie bevorzugen zudem automatisierte Korrekturen von fehlerhaftem Fahrverhalten. Fahrer mit einem eher riskanten „Driving Style“ bevorzugen Darstellungen, die das entsprechend riskantere Fahrerlebnis visualisieren können, beispielsweise geschwindigkeitsadaptive Lichtumgebungen im Fahrzeug [15]. Schrittweise Aufklärung über die Funktionsweisen automatisierter Fahrzeuge, die zu realistischeren mentalen Modellen über diese führen, verbessern ebenfalls Akzeptanz und Vertrauen [25], weiterhin auch die testweise Nutzung eines solchen Fahrzeugs [14]. Je zufriedener der Mensch zudem mit seinem eigenen Fahrstil ist, desto eher will er, dass das vollautomatisierte Fahrzeug seinen Stil adaptiert [14, 20].

In diesen Studien wird davon ausgegangen, dass die Einstellung zum Fahren sowie die Fahrfähigkeiten, die beim manuellen Fahren im Straßenverkehr gelten - also „Driving Style“ und „Driving Skill“ -, auch in hochautomatisierten Systemen weiterhin von Bedeutung sind. Um den veränderten Bedingungen Rechnung zu tragen, die sich mit der fortschreitenden Automatisierung von Fahrfunktionen ergeben, gilt es jedoch, die etablierten Typisierungen anzupassen und weiterzuentwickeln $[4,14,33]$ : So rückt die Rolle des menschlichen Fahrers auf den aktuell diskutierten Stufen der Automation (SAE-Level) stärker in Richtung eines Überwachers mit hohem Situationsbewusstsein, während die operativen Fahraufgaben an die Technik abgegeben werden [26]. Damit sind nicht mehr nur Fahrstil und -kompetenz im Umgang mit nicht-automatisierten Fahrzeugen von Relevanz, sondern auch Faktoren, die den Umgang und die Nutzung mit automatisierten Systemen prägen und die neuen Rolle des menschlichen Überwachers berücksichtigen. Die Kompetenzen im Umgang mit neuer Technik und die bisherige Erfahrung mit Fahrerassistenzsystemen (FAS) gewinnen damit an Bedeutung. Aufgrund der Automation müssen Fahrer neue Erfahrungen machen und ein Vertrauen gegenüber der Technik ausbilden [22, 33]. Die Akzeptanz einer solchen Technik ist darüber hinaus von Einstellungen gegenüber Innovationen und der Bereitschaft zur Abgabe von Kontrolle abhängig [37]. Um diesen Veränderungen bei der Fahrertypbestimmung Rechnung zu tragen, werden auf Basis bestehender Typisierungen aus der Verkehrspsychologie die Kategorien „Driving Skill“ und „Driving Style“ aufgegriffen und anhand von Erkenntnissen der Automationsforschung zum „Automated Driving Skill“ und „Automated Driving Style" weiterentwickelt. Methodisch werden dazu bestehende Messinstrumente mit weiteren relevanten Faktoren und Einflussvariablen ergänzt, die bislang nicht oder nur unzureichend in Typisierungen erforscht und erhoben wurden bzw. nun im Kontext des automatisierten Fahrens bedeutsam werden. 


\section{Methodisches Vorgehen}

\subsection{Entwicklung des Messinstruments und Operationalisierung}

Zur empirischen Erhebung des Fahrertyps (Driving Skill und Driving Style) konnten bisher zahlreiche etablierte Messinstrumente identifiziert werden $[6,16,23,34$, 36]. Die Instrumente unterscheiden sich hinsichtlich der inhaltlichen Schwerpunktsetzung, der Trennschärfe zwischen Driving Skill und Driving Style sowie der Anzahl an identifizierten Fahrertypen. Für die vorliegende Studie wurden Messinstrumente zum Driving Style und Driving Skill kombiniert und modifiziert sowie entsprechend der oben getroffenen Annahmen zur zunehmenden Automation ergänzt. Die Zuordnungen verstehen sich dabei als Heuristiken, die einen ersten Orientierungsrahmen entsprechend der vorherigen Unterscheidungsmerkmale zwischen „Driving Style“ und „Driving Skill“ bieten. Die Ergänzungen sind so zu verstehen, dass sie nicht mehr ausschließlich Einflussfaktoren berücksichtigen, die das manuelle Fahren prägen, sondern auch potenzielle Einflussfaktoren, welche das menschliche Verhalten im Umgang mit automatisierten Fahrsystemen - wie Technikaffinität, Vertrauen in automatisierte Systeme oder Adaptivitätsvorstellungen von Fahrassistenzsystemen - prägen.

Der finale Fragebogen beinhaltet neun Skalen sowie weitere Einzelfragen (z.B. zur Fahrerfahrung und zur Nutzung von FAS), die sich jeweils den beiden Oberkategorien „Automated Driving Style“ und „Automated Driving Skill“ zugeordnet wurden. Ergänzend wurden weiterhin demographische Merkmale (Alter, Geschlecht, Bildungsniveau) abgefragt. Zur Erhebung der Oberkategorie „Automated Driving Style" wurden folgende etablierte Konstrukte und Fragebögen eingesetzt, die sich in empirischen Studien hinsichtlich Reliabilität und Validität bewährt haben.

1. Der „Multidimensional Driving Style Inventory“ [34] misst spezifische Charakteristika des Fahrstils in Subskalen, u.a. aggressives, ängstliches, risikoreiches und vorsichtiges Fahren. Er dient der klassischen Erhebung des ,Driving Style“ und damit des klassischen Fahrertyps in der Einstellungsdimension.

2. Der Fragebogen „TA-EG“ [18] misst die allgemeine Technikaffinität in Bezug auf Begeisterung, Kompetenz sowie negativer und positiver Einstellung gegenüber Technik. Der zunehmenden Interaktion mit Technik sowie der veränderten Rollenverteilung zwischen Mensch und Technik beim automatisierten Fahren wird somit Rechnung getragen.

3. Gleiches gilt für die Skala "Trust in Automated Systems“ [17], die Vertrauen in ein bestimmtes technisches System (hier: selbstfahrendes Auto) bezüglich verschiedener
Faktoren wie Vertrautheit, Verlässlichkeit und Zuversicht erhebt.

4. Schließlich wurde die Kompatibilität automatisierter Technik zu eigener Lebenswelt bzw. die erwartete Veränderung gegenüber der bisherigen Erfahrung (Vorerfahrungen, Wertevorstellungen, eigener Mobilität) mittels einer Skala innerhalb des „Automation Acceptance Model" [9] gemessen.

Zur Erhebung der Oberkategorie „Automated Driving Skill“ wurden folgende Konstrukte und Fragebögen eingesetzt:

1. Zur Erfassung des klassischen Driving Skill wurde das „Driver Stress Inventory“ [24] verwendet. Dieses misst abweichendes Fahrverhalten in konkreten, zum Teil kritischen Verkehrssituationen.

2. Ergänzt wurde dieses durch den „Driver Coping Questionnaire“ [24], welcher das situative Bewältigungsverhalten von Fahrern im Straßenverkehr, also den Umgang und die Handlungsmuster als Reaktionen auf bestimmte (kritische) Situationen misst.

3. Eine große Herausforderung stellt die quantitative Vorab-Messung eines mentalen Modells von Fahrern in Bezug auf Fahrassistenzsysteme und automatisiertes Fahren dar. Das Modell repräsentiert die Vorstellung, wie ein (technisches) System funktioniert bzw. welche Funktionen es hat. Das mentale Modell beeinflusst damit direkt die Art und Weise, wie ein System genutzt wird. Um ein allgemeines und vereinfachtes, durchschnittliches mentales Modell zum teilautomatisierten Fahren zu erheben, sollten die Probanden ein bestimmtes Fahrassistenzsystem hinsichtlich wahrgenommener oder vermuteter Anpassbarkeit an den eigenen Fahrstil (Adaptivität), Komfort und Sicherheit bewerten [2]. Die Wahl des zu bewertenden FAS fiel auf das ACC (Adaptive Cruise Control), da es essenzielle Fahraufgaben im Rahmen des teilautomatisierten Fahrens übernimmt ${ }^{1}$.

\subsection{Stichprobe und Gütemessungen}

Die Umfrage wurde im Januar 2019 über einen Dienstleister durchgeführt. Es wurden nur Teilnehmer für die Studie akquiriert, die in Deutschland leben und einen Führerschein besitzen. Der Datensatz bestand ursprünglich aus 1688 Fällen. Entfernt wurden unvollständige Fälle und Fälle, mit inkorrekt beantworteten Kontrollfragen. Damit verbleiben

\footnotetext{
${ }^{1}$ Innerhalb des Fragebogens wurde dieser Themenbereich zu Beginn abgefragt und stellt damit das Framing für alle weiteren Fragen bezüglich des automatisierten Fahrens dar. Dazu wurden eine grafische Veranschaulichung des ACC bereitgestellt sowie automatisierte Fahrfunktionen des ACC in einem kurzen Text erläutert.
} 
1420 Fälle $(84,12 \%)$. Die Repräsentativität wurde durch Quoten auf die demographischen Merkmale Alter (Durchschnitt: 47,6 Jahre) und Geschlecht (47,7\% weiblich) gewährleistet. Menschen mit höherem Bildungsabschluss sind in dieser Stichprobe jedoch leicht überrepräsentiert $(31,2 \%$ mit Hochschulabschluss).

Die Güteprüfung der Faktoren erfolgte in zwei Schritten: Zur Dimensionsreduktion wurden Faktorenanalysen zu den jeweiligen Konstrukten durchgeführt. Items mit hohen Nebenladungen wurden entfernt. Die so strukturierten Faktoren wurden dann mittels der Reliabilitätsanalyse auf ihre interne Konsistenz geprüft. Die erwarteten Faktoren konnten so plausibel abgebildet werden. Cronbach's Alpha-
Werte (Abk. CA) über 0,7 gelten als gut für die weitere Durchführung. Dieses Kriterium ist für alle gebildeten Faktoren erfüllt. Anschließend wurde mittels konfirmatorischer Faktorenanalyse die Güte der Faktoren ${ }^{2}$ bestimmt. Die globalen Fit-Indizes weisen auf einen akzeptablen bis guten Fit der Faktorenmodellierung hin: $\chi 2 / \mathrm{df}=3,05$; RMSEA =0,038; SRMR=0,060; CFI=0,906.

Für alle Faktoren übersteigt der Wert der Konstruktreliabilität (Abk. KR) den typischerweise angenommenen Grenzwert von 0,6. Die durchschnittlich extrahierte Varianz (Abk. AVE) liegt bei den Faktoren ,Selfmonitoring im Straßenverkehr“ und „Aggression im Straßenverkehr“ weit unter dem geforderten Grenzwert von 0,5. Beide Faktoren

Tab. 1 Verwendete Skalen und erhobene Merkmale

\begin{tabular}{|c|c|c|c|c|c|}
\hline Messinstrument & Items & Faktoren & $\mathrm{CA}$ & $\mathrm{KR}$ & AVE \\
\hline \multicolumn{6}{|c|}{ Automated Driving Style ${ }^{\mathrm{a}}$} \\
\hline \multirow{4}{*}{$\begin{array}{l}\text { Multidimensional } \\
\text { Driving Style } \\
\text { Inventory [34] }\end{array}$} & 4 & Aggressives Fahrverhalten (Angry) & 0,741 & 0,716 & 0,588 \\
\hline & 3 & Risikoreiches Fahrverhalten (Risky) & 0,844 & 0,846 & 0,648 \\
\hline & 2 & Vorsichtiges Fahrverhalten (Careful) & 0,818 & 0,820 & 0,695 \\
\hline & 4 & Aversion gegenüber Fahren (Aversion) & 0,830 & 0,828 & 0,554 \\
\hline \multirow{2}{*}{$\begin{array}{l}\text { Technikaffinität (TA- } \\
\text { EG) [18] }\end{array}$} & 4 & Begeisterung für Technik & 0,790 & 0,902 & 0,698 \\
\hline & 4 & Kompetenz im Umgang mit Technik & 0,906 & 0,740 & 0,428 \\
\hline $\begin{array}{l}\text { Trust in Automated } \\
\text { Systems [17] }\end{array}$ & 5 & Vertrauen in automatisierte Systeme & 0,828 & 0,811 & 0,427 \\
\hline \multirow{3}{*}{$\begin{array}{l}\text { Compatibility } \\
\text { (aus Automation } \\
\text { Acceptance Model) [9] }\end{array}$} & 3 & Kompatibilität mit bisherigen Erfahrungen & 0,819 & 0,790 & 0,558 \\
\hline & 3 & Kompatibilität mit eigenen Wertvorstellungen & 0,744 & 0,700 & 0,445 \\
\hline & 3 & Kompatibilität mit eigener Mobilität ${ }^{\mathrm{d}}$ & 0,740 & 0,766 & 0,540 \\
\hline \multicolumn{6}{|c|}{ Automated Driving Skill ${ }^{\mathrm{a}}$} \\
\hline \multirow{4}{*}{$\begin{array}{l}\text { Driver Stress Inventory } \\
{[24]}\end{array}$} & 5 & Aufmerksamkeit im Straßenverkehr & 0,839 & 0,835 & 0,506 \\
\hline & 6 & Selfmonitoring im Straßenverkehr ${ }^{c}$ & 0,800 & 0,789 & 0,387 \\
\hline & 5 & Aggression im Straßenverkehr ${ }^{c}$ & 0,778 & 0,761 & 0,392 \\
\hline & 3 & Müdigkeit im Straßenverkehr ${ }^{\text {d }}$ & 0,832 & 0,792 & 0,561 \\
\hline \multirow{4}{*}{$\begin{array}{l}\text { Driver Coping } \\
\text { Questionnaire [24] }\end{array}$} & 3 & Reflexionsfähigkeit über das eigene Fahrverhalten & 0,828 & 0,822 & 0,606 \\
\hline & 4 & Situationsbewusstsein im Straßenverkehr & 0,803 & 0,807 & 0,512 \\
\hline & 4 & Verhaltenskontrolle im Straßenverkehr & 0,840 & 0,846 & 0,581 \\
\hline & 3 & Lerneffekte bei kritischen Situationen $^{\mathrm{d}}$ & 0,738 & 0,747 & 0,498 \\
\hline \multirow{3}{*}{$\begin{array}{l}\text { Mentales Modell } \\
\text { (FAS- } \\
\text { Systembewertung) [2] }\end{array}$} & 4 & Sicherheitsvorstellung bezügl. FAS & 0,859 & 0,871 & 0,633 \\
\hline & 4 & Adaptivitätsvorstellung bezügl. FAS & 0,856 & 0,725 & 0,470 \\
\hline & 3 & Komfortvorstellungen bezügl. FAS & 0,777 & 0,838 & 0,566 \\
\hline \multicolumn{6}{|c|}{ Demografische und sonstige Merkmale ${ }^{\mathrm{d}}$} \\
\hline $\begin{array}{l}\text { Demografische Merk- } \\
\text { male }\end{array}$ & - & Alter, Geschlecht, Bildungsgrad & - & - & - \\
\hline Fahrerfahrung & 1 & Durchschnittlich gefahrener Kilometer pro Jahr & - & - & - \\
\hline $\begin{array}{l}\text { Nutzungshäufigkeit } \\
\text { von FAS }\end{array}$ & 4 & $\begin{array}{l}\text { Tempomat/Geschwindigkeitsassistent, Spurhalteassistent/ } \\
\text { Spurverlassenswarner, Spurwechselassistent, ACC }\end{array}$ & - & - & - \\
\hline
\end{tabular}

CA Cronbachs' Alpha, KR Konstruktreliabilität, AVE Average variance extracted

${ }^{a}$ Items wurden auf einer Skala von 1 bis 5 erhoben, niedrige Werte entsprechen dabei einer geringen Merkmalsausprägung

${ }^{\mathrm{b}}$ erhoben auf einer Skala von $0=$ keine Verwendung bis $5=$ (fast) immer verwendet

${ }^{\mathrm{c}}$ aufgrund geringer Güte für Clusteranalyse verworfen

$\mathrm{d}$ aufgrund mangelndem Beitrag zur Differenzierung für Clusteranalyse verworfen

2 Typischerweise akzeptierte Werte für die einzelnen Gütekriterien finden sich z. B. bei Hair et al. [13]. 
wurden daher nicht weiter berücksichtigt. Für die Faktoren „Vertrauen in automatisierte Systeme“ und „Adaptivitätsvorstellung bezügl. FAS“ liegen die Werte leicht unter 0,5. Jedoch übersteigt die AVE die quadrierten Korrelationen zu allen anderen Faktoren (Fornell-Larcker-Kriterium) [8]. Da weiterhin alle anderen Gütekriterien erfüllt sind (siehe Tab. 1), wurden beide Faktoren daher beibehalten. Ähnliches gilt für die Faktoren „Aufmerksamkeit im Straßenverkehr“ und „Situationsbewusstsein im Straßenverkehr" sowie für die Faktoren „Begeisterung für Technik“ und „Kompetenz im Umgang mit Technik“. Nur zwischen diesen Faktorenpaaren übersteigt die quadrierte Korrelation jeweils die AVE. Die vier Faktoren wurden daher und aufgrund dieser geringen Abweichung sowie ihrer theoretischen Bedeutung beibehalten. Alle weiteren Faktoren erfüllen das Fornell-Larcker-Kriterium vollständig ${ }^{3}$.

Die in Tab. 1 aufgelisteten Ergebnisse zeigen die Konstrukte, die verbliebene Anzahl relevanter Items sowie die Gütekriterien für die Faktoren. Die verbliebenen Faktoren wurden für die Clusteranalyse aufgenommen. Dabei zeigte sich, dass die Faktoren „Kompatibilität mit eigener Mobilität“, „Müdigkeit im Straßenverkehr“, und „Lerneffekte bei kritischen Situationen“ zu keiner oder nur einer sehr geringen Differenzierung beitragen. Diese Faktoren wurden daher ebenfalls entfernt und die Clusteranalyse wiederholt durchgeführt.

\section{Analyse und Ergebnisse}

\subsection{Clusterbeschreibung}

Zur Identifikation der neuen Fahrertypen wurde eine TwoStep-Clusteranalyse durchgeführt. Diese erlaubt es, sowohl kategoriale als auch stetige Variablen in großen Datensätzen zu berücksichtigen. Es entstand eine Drei-Cluster-Lösung, bei denen die Cluster ähnlich verteilt waren (siehe Tab. 2). Die Mittelwertunterschiede zwischen Clustern wurden mittels ANOVA und Post-Hoc-Tests geprüft.

Im Cluster A finden sich Umfrageteilnehmer, die ein hohes generelles Vertrauen in Technik und Automation, eine hohe Technikaffinität und hohe Kompatibilitätswerte bezüglich des automatisierten Fahrens mit den eigenen Wertevorstellungen und dem Erfahrungshorizont vorweisen (der „Zuversichtliche“). Gleichzeitig besitzen sie ein mentales Modell des teilautomatisierten Fahrens, das FAS mit hoher Sicherheit, starker Adaptivität und großem Komfort assoziiert. Der Driving Style lässt sich als weniger riskant und aggressiv, eher vorsichtig beschreiben. Es liegt gleichzei-

\footnotetext{
${ }^{3}$ In der späteren Clusteranalyse erwiesen sich diese Faktoren als gewinnbringend für die Differenzierung der Cluster. Dies bestätigt die Entscheidung zur Beibehaltung der Faktoren.
}

tig eine niedrige Aversion gegen das Autofahren vor. Die Gruppe schätzt sich als sehr aufmerksam, situationsbewusst und kontrolliert im Straßenverkehr ein.

Personen im Cluster B sind von einer niedrigeren Aufgeschlossenheit gegenüber dem automatisierten Fahren (der „Überforderte“) geprägt. Das Vertrauen in automatisierte Systeme ist geringer, gleichzeitig ist es nicht kompatibel zu ihren Wertevorstellungen. Auch gehen sie davon aus, dass automatisiertes Fahren kaum neue Erfahrungen mit sich bringen wird. Daher wird die vorhandene negative Grundeinstellung gegenüber dem Fahren beibehalten. Die Gruppe zeichnet sich durch eine niedrige allgemeine Technikaffinität aus und besitzt ein mentales Modell vom teilautomatisierten Fahren, das insbesondere skeptisch bei der Adaptivität des Systems ist. Ähnlich zu Cluster A schätzen die Personen in Cluster B ihren Fahrstil als risikoarm und wenig aggressiv, sondern eher als vorsichtig ein. Es liegt eine höhere Aversion gegenüber dem Fahren vor. Die Teilnehmer halten sich für aufmerksam im Straßenverkehr und bemühen sich um einen besonnenen Fahrstil.

Im Cluster $\mathrm{C}$ finden sich Probanden mit einem laut Selbsteinschätzung gegenüber den beiden anderen Gruppen vergleichsweise risikoreichen, aggressiven und wenig vorsichtigen Fahrstil (der „Selbstbewusste“). Werte zu Vertrauen gegenüber dem und Kompatibilität der Wertevorstellungen mit dem automatisierten Fahren sowie allgemein zur Technikaffinität liegen im mittleren Bereich. Die Gruppe schätzt die Nutzung automatisierter Fahrzeuge gegenüber den anderen Teilnehmern nochmal geringer als neue (Fahr-)Erfahrung ein. Die Personen im Cluster C halten sich im Straßenverkehr zudem für weniger aufmerksam als die Personen in den anderen Clustern, reflektieren ihr Fahrverhalten allerdings mehr. Sie weisen eine geringere Verhaltenskontrolle auf und neigen somit stärker zu Konfrontationen mit anderen Verkehrsteilnehmern. Ihr mentales Modell ist von einer mittleren angenommenen Sicherheit und Adaptivität sowie vergleichsweise niedriger Komfortvorstellung gegenüber dem (teil)automatisierten Fahren geprägt.

\subsection{Fahrertypen im Fahrsimulator}

In einem experimentellen Setting wurde überprüft, inwiefern die repräsentativ ermittelten Fahrertypen unterschiedliche Verhaltensweisen und Einstellungen bei und nach Testfahrten mit automatisierten Fahrzeugen im Simulator zeigen. Mittels einer Kurz-Version ${ }^{4}$ des oben vorgestellten Fragebogens konnten vor Beginn des Fahrversuchs die Probanden einem der drei Cluster zugeordnet werden. Für die Ana-

\footnotetext{
${ }^{4}$ Um die höchste Übereinstimmung zwischen der originalen Version und der Kurzversion des Fragebogens zu erzielen, wurden diejenigen Items mit der höchsten Faktorladung in die Kurzversion aufgenommen.
} 
Tab. 2 Clusterbeschreibung

\begin{tabular}{|c|c|c|c|c|c|c|}
\hline \multirow{2}{*}{$\begin{array}{l}\text { Merkmale } \\
\text { Deskriptive Statistiken }\end{array}$} & \multicolumn{2}{|c|}{ Cluster A „Zuversichtliche“ } & \multicolumn{2}{|c|}{ Cluster B „Überforderte“ } & \multicolumn{2}{|c|}{ Cluster C „Selbstbewusste“ } \\
\hline & & & & & & \\
\hline Relative Größe & \multicolumn{2}{|c|}{$35,8 \%(n=508)$} & \multicolumn{2}{|c|}{$31,0 \%(n=440)$} & \multicolumn{2}{|c|}{$33,2 \%(n=472)$} \\
\hline Geschlecht & \multicolumn{2}{|c|}{$\begin{array}{l}\mathrm{w}=42,5 \% \\
\mathrm{~m}=57,5 \%\end{array}$} & \multicolumn{2}{|c|}{$\begin{array}{l}\mathrm{w}=55,2 \% \\
\mathrm{~m}=44,8 \%\end{array}$} & \multicolumn{2}{|c|}{$\begin{array}{l}\mathrm{w}=46,2 \% \\
\mathrm{~m}=53,8 \%\end{array}$} \\
\hline Alter & \multicolumn{2}{|c|}{ 43,3 Jahre } & \multicolumn{2}{|c|}{ 50,9 Jahre } & \multicolumn{2}{|c|}{ 48,6 Jahren } \\
\hline Fahrerfahrung & \multicolumn{2}{|l|}{$11.000 \mathrm{~km}$} & \multicolumn{2}{|c|}{$9280 \mathrm{~km}$} & \multicolumn{2}{|c|}{$10.240 \mathrm{~km}$} \\
\hline Nutzungshäufigkeit von FAS & \multicolumn{2}{|l|}{1,21} & \multicolumn{2}{|l|}{0,73} & \multicolumn{2}{|c|}{1,35} \\
\hline \multicolumn{7}{|l|}{ Automated Driving Style (Mean/STD) } \\
\hline Aggressives Fahrverhalten (Angry) & $2,31^{\mathrm{C}}$ & 0,83 & $2,33^{\mathrm{C}}$ & 0,79 & $3,01^{\mathrm{AB}}$ & 0,71 \\
\hline Risikoreiches Fahrverhalten (Risky) & $1,35^{\mathrm{C}}$ & 0,52 & $1,27^{\mathrm{C}}$ & 0,48 & $2,38^{\mathrm{AB}}$ & 0,93 \\
\hline Vorsichtiges Fahrverhalten (Careful) & $4,05^{\mathrm{C}}$ & 0,79 & $3,96^{\mathrm{C}}$ & 0,85 & $3,32^{\mathrm{AB}}$ & 0,75 \\
\hline Aversion gegenüber Fahren (Aversion) & $1,80^{\mathrm{BC}}$ & 0,79 & $2,47^{\mathrm{AC}}$ & 0,99 & $2,23^{\mathrm{AB}}$ & 0,74 \\
\hline Begeisterung für Technik & $3,90^{\mathrm{BC}}$ & 0,81 & $2,69^{\mathrm{AC}}$ & 0,98 & $3,42^{\mathrm{AB}}$ & 0,81 \\
\hline Kompetenz im Umgang mit Technik & $4,18^{\mathrm{BC}}$ & 0,64 & $3,41^{\mathrm{A}}$ & 0,87 & $3,52^{\mathrm{A}}$ & 0,69 \\
\hline Vertrauen in automatisierte Systeme & $3,28^{\mathrm{BC}}$ & 0,74 & $2,03^{\mathrm{AC}}$ & 0,64 & $2,97^{\mathrm{AB}}$ & 0,51 \\
\hline $\begin{array}{l}\text { Kompatibilität mit bisherigen Erfah- } \\
\text { rungen }\end{array}$ & $1,53^{\mathrm{BC}}$ & 0,60 & $2,18^{\mathrm{AC}}$ & 0,93 & $2,53^{\mathrm{AB}}$ & 0,75 \\
\hline $\begin{array}{l}\text { Kompatibilität mit eigenen Wertvor- } \\
\text { stellungen }\end{array}$ & $3,60^{\mathrm{BC}}$ & 0,86 & $2,10^{\mathrm{AC}}$ & 0,85 & $2,98^{\mathrm{AB}}$ & 0,62 \\
\hline \multicolumn{7}{|l|}{ Automated Driving Skill (Mean/STD) } \\
\hline Aufmerksamkeit im Straßenverkehr & $4,42^{\mathrm{BC}}$ & 0,47 & $4,17^{\mathrm{AC}}$ & 0,58 & $3,52^{\mathrm{AB}}$ & 0,50 \\
\hline $\begin{array}{l}\text { Reflexionsfähigkeit über das eigene } \\
\text { Fahrverhalten }\end{array}$ & $2,22^{\mathrm{C}}$ & 0,91 & $2,11^{\mathrm{C}}$ & 0,93 & $2,76^{\mathrm{AB}}$ & 0,74 \\
\hline $\begin{array}{l}\text { Situationsbewusstsein im Straßenver- } \\
\text { kehr }\end{array}$ & $4,41^{\mathrm{BC}}$ & 0,50 & $4,17^{\mathrm{AC}}$ & 0,61 & $3,41^{\mathrm{AB}}$ & 0,56 \\
\hline Verhaltenskontrolle im Straßenverkehr & $4,09^{\mathrm{C}}$ & 0,75 & $4,06^{\mathrm{C}}$ & 0,72 & $3,19^{\mathrm{AB}}$ & 0,66 \\
\hline Sicherheitsvorstellung bzgl. FAS & $4,63^{\mathrm{BC}}$ & 0,44 & $3,71^{\mathrm{AC}}$ & 0,78 & $3,83^{\mathrm{BC}}$ & 0,63 \\
\hline Adaptivitätsvorstellung bzgl. FAS & $3,70^{\mathrm{BC}}$ & 0,73 & $2,87^{\mathrm{AC}}$ & 0,64 & $3,34^{\mathrm{BC}}$ & 0,57 \\
\hline Komfortvorstellungen bzgl. FAS & $4,14^{\mathrm{BC}}$ & 0,74 & $3,23^{\mathrm{A}}$ & 0,83 & $3,12^{\mathrm{A}}$ & 0,73 \\
\hline
\end{tabular}

$A-C$ signifikanter Unterschied zu dem jeweiligen Cluster auf dem Signifikanzniveau von $p \leq 0,01$

lyse werden die Daten von 36 Teilnehmern herangezogen. Dazu wurden für jeden Faktor die euklidischen Distanzen zwischen den Ergebnissen der Selbsteinschätzung durch den Fragebogen zu den entsprechenden Clusterzentren aus der Online-Studie ermittelt. Der Cluster, zu dem insgesamt die Distanzen am geringsten sind, gibt den Fahrertyp vor (hard clustering). Von den 36 Probanden entsprechen 23 dem Typ „Zuversichtliche“ (Cluster A), fünf dem Typ „Überforderte“ (Cluster B), und acht dem Typ ,Selbstbewusste“ (Cluster C). Damit ist der „Zuversichtliche“ in dieser Studie überrepräsentiert, da er einfacher für Studien zum automatisierten Fahren zu rekrutieren ist.

Die Probanden durchliefen eine 20-minütige Testfahrt im Fahrsimulator sowie anschließend verschiedene Fahrszenarien (Schnee, Nebel, Nacht, Autobahn, Landstraße etc.). Das Fahrzeug in der Simulation war mit ACC und Spurhalteassistent ausgestattet, die je nach Szenario von den Probanden eingesetzt, überwacht oder übersteuert werden mussten. Nach dem gesamten Fahrversuch wurden das Fahrerlebnis sowie die Erfahrungen mit den FAS evalu- iert (Tab. 3): In Anlehnung an den NASA-TLX (NASATask Activity Load Index) bzw. dessen adaptierte Variante DALI (Driving Activity Load Index; [20]) wurden der subjektiv empfundene Stress und die wahrgenommene Kontrolle erhoben. Ergänzend wurde die Zufriedenheit mit der Aufgabenverteilung erfasst [1]. Angelehnt an das bereits erwähnte Automation Acceptance Model [9] wurden die Einfachheit der Nutzung und die wahrgenommene Nützlichkeit erhoben. Letzteres wurde auf die Entlastung beim Fahren (Sicherheit, Flexibilität) ausgerichtet. Zudem wurden die Nutzungsabsicht und die Kompatibilität mit dem eigenen Mobilitätsverhalten vor und nach der Simulation erhoben, um Veränderungen durch den tatsächlichen Umgang mit FAS zu bewerten. Um die Ergebnisse im Simulator hinsichtlich der Übertragbarkeit einzuschätzen, gaben die Probanden am Ende des Experiments ihre Einschätzung hinsichtlich des Realismusempfindens bezüglich des realen Fahrens sowie bezüglich der eigenen Fahrweise ab. Für beide Aspekte bewerteten alle drei Fahrertypen das Realismusempfinden als akzeptabel bis hoch. Es ist daher 
Tab. 3 Ergebnisse der Simulatorstudie nach Fahrertypen

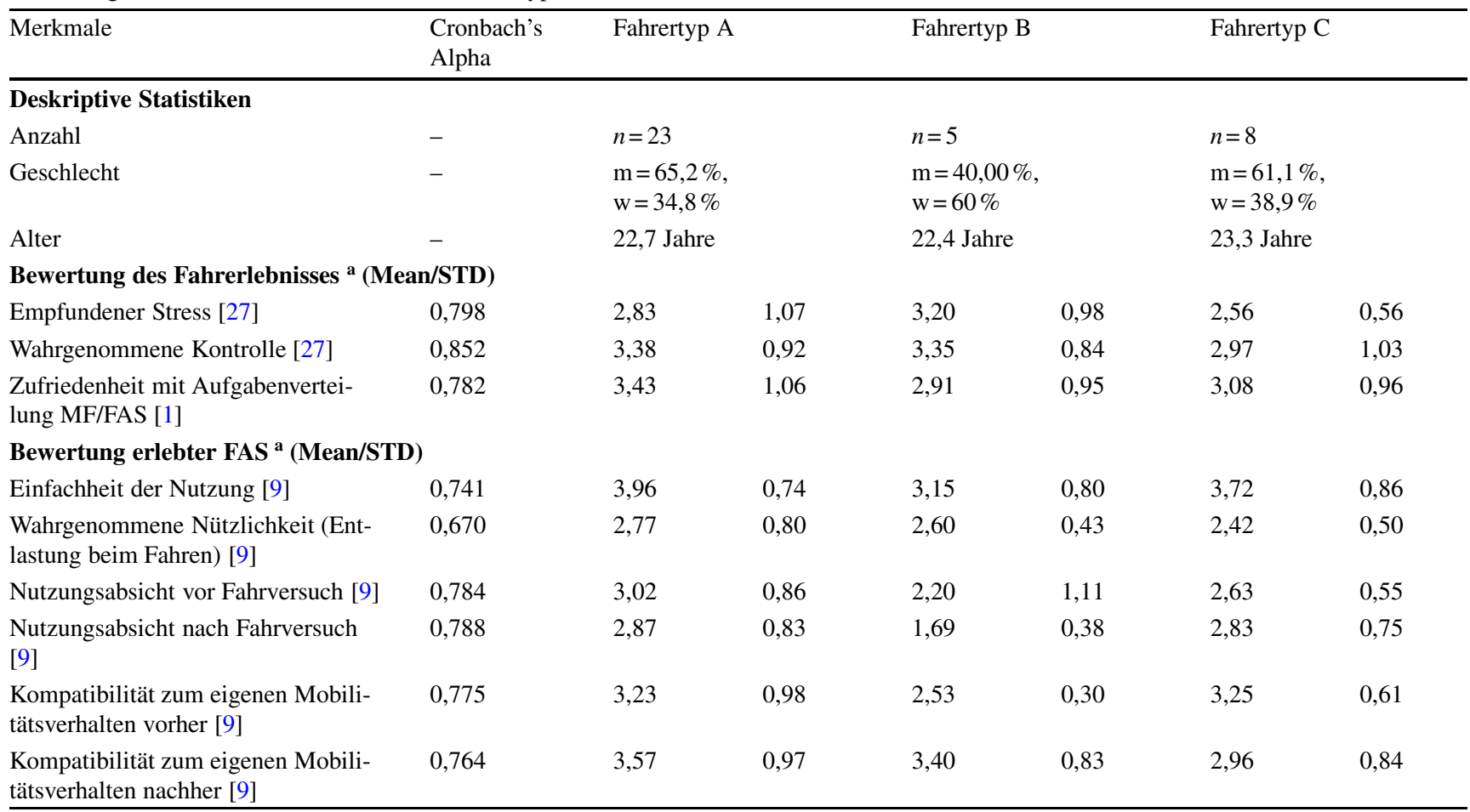

${ }^{a}$ Items wurden auf einer Skala von 1 bis 5 erhoben, niedrige Werte entsprechen dabei einer geringen Merkmalsausprägung; aufgrund der geringen Stichprobe, wurde auf die Angabe von Signifikanzen verzichtet

anzunehmen, dass der Simulator reales Fahren angemessen abbilden kann.

Mit seinen zumeist positiven Bewertungen von und Einstellungen gegenüber den FAS und den Übergabeprozessen vermittelt der ,zuversichtliche“ Fahrertyp das erwartete optimistische Bild zum automatisierten Fahren. Insbesondere fällt auf, dass sowohl die Nutzungsabsicht von FAS generell als auch die Nützlichkeit der spezifisch in den Szenarien genutzten FAS positiv bewertet werden. Die subjektiv bewertete Kompatibilität von FAS mit dem eigenen Mobilitätsverhalten wurden vor dem Versuch als überdurchschnittlich hoch und nach dem Fahrversuch als nochmals höher eingeschätzt, während die Nutzungsabsicht auf hohem Niveau gleichblieb. Dieser Fahrertyp bewertete die Nützlichkeit sowie die Einfachheit der Nutzung der FAS am höchsten von allen Fahrertypen. Das im Simulator erlebte automatisierte Fahren entsprach somit größtenteils den positiven Erwartungen. Es kann somit interpretiert werden, dass das Vertrauen in die FAS hoch und die Bereitschaft, die Kontrolle an die automatisierten Systeme abzugeben, hoch sind.

Der „Überforderte“ bewertet die Nützlichkeit der FAS deutlich negativer als die anderen beiden Fahrertypen; auch die Werte für die Nutzungsabsicht sind niedriger. Er zeigt eine im Vergleich geringe Zufriedenheit mit der Verteilung der Fahraufgaben zwischen Mensch und Technik. Dies ist konsistent mit den Evaluationen zum empfundenen hohen Stress trotz hoher Automation im Fahrversuch. Dies zeigt eine tendenziell tatsächlich vorhandene Überforderung dieses Fahrertyps auf. Allerdings wird die Kompatibilität der FAS mit dem eigenen Mobilitätsverhalten nach dem Fahrversuch deutlich höher bewertet als vorher. Dies lässt vermuten, dass dieser Fahrertyp bestehende Akzeptanzbarrieren ablegen kann, dass aber die spezifisch in diesem Experiment vorhandenen Handlungsmuster der FAS nicht zu einer höheren Akzeptanz oder Nutzungsabsicht geführt haben. Eine höhere Akzeptanz von FAS bzw. Übergabeprozessen kann womöglich nur durch weitergehende Änderungen erreicht werden, beispielsweise eine noch stärkere Automation, in der der Fahrer keine Überwachungstätigkeiten mehr ausführen müsste.

Beim „Selbstbewussten“ ergibt sich ein zweigeteiltes Bild: Während die von den Fahrversuchen unabhängige Bewertung zur Nutzungsabsicht von FAS hoch ist, wird die in den Fahrversuchen wahrgenommene Nützlichkeit und Einfachheit der genutzten FAS als niedrig im Vergleich zu den anderen Fahrertypen bewertet. Dies lässt vermuten, dass der Selbstbewusste zwar generell eher bereit ist, FAS zu nutzen, aber nicht in der Art und Weise, wie sie im Fahrversuch zu erleben waren. Auch wurde die Kompatibilität der FAS zum eigenen Mobilitätsverhalten nach den Fahrversuchen als deutlich niedriger gegenüber vorher evaluiert. Ein Grund für diese Ergebnisse kann die als geringer eingeschätzte wahrgenommene Kontrolle sein, die der Fahrertyp während der Nutzung der FAS empfand. 
Da dieser Fahrertyp hohen Fahrspaß empfindet und gern die Autorität über sein Fahrzeug besitzt, hat ihm der Fahrversuch womöglich zuvor nicht offensichtliche Nachteile aufgezeigt, die mit seinem Fahrverhalten inkongruent sind.

\section{Fazit und Ausblick}

Das Ziel der Studie war es, Fahrertypen im Kontext des automatisierten Fahrens zu identifizieren. Dazu wurde zunächst ein Online-Fragebogen auf Basis etablierter Skalen zur Fahrertypisierung im Straßenverkehr erstellt. Der Fragebogen wurde um weitere Skalen aus den Bereichen der Automatisierungsforschung und Technikakzeptanz ergänzt, um die klassischen Typisierungsansätze um eine Komponente zum automatisierten Fahren zu erweitern. Aus den Ergebnissen der repräsentativen Online-Befragung wurden mittels einer Clusteranalyse drei Fahrertypen identifiziert. Diese unterscheiden sich jeweils in ihren Fahrstilen, insbesondere aber auch in ihrer Einstellung gegenüber dem automatisierten Fahren: Der „Zuversichtliche“ blickt optimistisch auf das automatisierte Fahren und erwartet einen positiven Nutzen hinsichtlich Komfort, Sicherheit und Adaptivität. Der „Überforderte“ ist demgegenüber ablehnend, da er von einer weiteren Automatisierung weniger positive Wirkungen auf das Fahrerleben erwartet. Der „Selbstbewusste" zeigt hier eine ambivalente Haltung. Hier besteht anscheinend Unsicherheit darüber, ob zunehmende Technik im Fahrzeug sein gewohntes Fahrverhalten beeinträchtigt oder aber bereichert. Mit Hilfe der Fahrsimulator-Studie wurde dargestellt, dass diese Typen auch in realitätsnahen Verkehrssituationen unterschiedliche Einstellungen und Verhaltensweisen bezüglich des automatisierten Fahrens zeigen. Insbesondere ergaben sich Differenzen beim wahrgenommenen Stress und der Einfachheit der Nutzung der FAS. In einem nächsten Schritt kann die hier vorgeschlagene Typisierung dazu genutzt werden, Fragen zur konkreten adaptiven Technikgestaltung zu erforschen.

In bisherigen Studien wurde gezeigt, dass die Akzeptanz automatisierter Fahrsysteme von der eigenen Einstellung zum Fahren sowie der Fahrkompetenz abhängen. Die hier vorgeschlagene Erweiterung bestehender Typisierungen zu den Kategorien „Automated Driving Style“ und „Automated Driving Skill“ zeigt dabei auf, dass diese Akzeptanzunterschiede nicht nur aufgrund der klassischen Variablen des „Driving Skill“ und „Driving Style“ bestehen, sondern auch aufgrund von Faktoren wie Technikaffinität, Vertrauen in automatisierte Systeme sowie Sicherheits- und Adaptivitätsvorstellungen von FAS. Wir plädieren daher dafür, die in den beiden Kategorien ergänzten Faktoren wie Technikaffinität bei der zukünftigen Gestaltung automatisierter Fahrzeugtechnik zu berücksichtigen. Aus den Studienergebnissen lässt sich schließen, dass die hier identi- fizierten Fahrertypen unterschiedlich skeptisch und offen gegenüber der Automatisierung von Fahraufgaben sind und diese Einstellungen bei der Entwicklung adaptiver Automatisierungstechnologien im Fahrzeug berücksichtigt werden sollten. Die hier vorgeschlagenen Oberkategorien des „Automated Driving Skill“ und „Automated Driving Style“ sollten in weiteren Studien empirisch validiert werden. Dabei könnten in quantitativen Untersuchungen die Güte der Faktoren weiter gesteigert werden sowie die Korrelationen zwischen den Faktoren stärker berücksichtigt werden. In der hier durchgeführten Studie wurden zudem Überschneidungen zwischen den Clustern bezüglich einzelner Faktoren festgestellt. Weiter Studien könnten hier zu einer schärferen Trennung beitragen. Zudem können qualitative Methoden genutzt werden, um bislang nicht bekannte Einflussfaktoren für die Akzeptanz des automatisierten Fahrens aufzudecken.

Auch die experimentelle Studie unterliegt Limitationen, da reale Fahrsituationen nur simuliert wurden und dort die Komplexität des realen Straßenverkehrs nicht vollständig abgebildet werden kann. Weiterhin lag eine unausgeglichene Anzahl an Fahrertypen vor; es wurde eine kleine, vorrangig junge Teilnehmergruppe untersucht. Die Ergebnisse konnten daher nicht auf Signifikanzen hin untersucht werden. In einer Anschlussforschung sollte daher berücksichtigt werden, dass ausreichend viele Studienteilnehmer zu den drei Fahrertypen vorliegen. Die Typisierung basiert zudem alleine auf Selbstauskünften der befragten Personen. Ein Abgleich mit Beobachtungen von außen oder objektiven Messungen könnte dazu beitragen, die externe Validität der Typisierung nochmals zu stärken. Darüber hinaus wurde eine eindeutige Zuordnung zu den Fahrertypen (hard clustering) vorgenommen. Da in realen Verkehrssituationen Mischtypen existieren können, wäre in anschließenden Studien eine relative Zuordnung der Fahrertypen (soft clustering) sinnvoll. Die hier präsentierten Ergebnisse geben Anlass, die Forschung zu Fahrertypen im Kontext des automatisierten Fahrens weiter zu verfolgen, um die nutzergerechte Entwicklung des automatisierten Fahrens zu unterstützen.

Funding This research was supported by the Federal Ministry of Transport and Digital Infrastructure, Germany.

Die Forschung wurde vom Bundesministerium für Verkehr und digitale Infrastruktur unterstützt.

Funding Open Access funding enabled and organized by Projekt DEAL.

Interessenkonflikt J. Schlüter, M. Hellmann und J. Weyer geben an, dass kein Interessenkonflikt besteht.

The authors of this manuscript certify that they have NO affiliations with or involvement in any organization or entity with any financial interest or non-financial interest in the subject matter or materials discussed in this manuscript. 
Open Access Dieser Artikel wird unter der Creative Commons Namensnennung 4.0 International Lizenz veröffentlicht, welche die Nutzung, Vervielfältigung, Bearbeitung, Verbreitung und Wiedergabe in jeglichem Medium und Format erlaubt, sofern Sie den/die ursprünglichen Autor(en) und die Quelle ordnungsgemäß nennen, einen Link zur Creative Commons Lizenz beifügen und angeben, ob Änderungen vorgenommen wurden.

Die in diesem Artikel enthaltenen Bilder und sonstiges Drittmaterial unterliegen ebenfalls der genannten Creative Commons Lizenz, sofern sich aus der Abbildungslegende nichts anderes ergibt. Sofern das betreffende Material nicht unter der genannten Creative Commons Lizenz steht und die betreffende Handlung nicht nach gesetzlichen Vorschriften erlaubt ist, ist für die oben aufgeführten Weiterverwendungen des Materials die Einwilligung des jeweiligen Rechteinhabers einzuholen.

Weitere Details zur Lizenz entnehmen Sie bitte der Lizenzinformation auf http://creativecommons.org/licenses/by/4.0/deed.de.

\section{Literatur}

1. Altendorf E, Schreck C, Flemisch F (2017) A new method and results for analyzing decision-making processes in automated driving on highways. In: Stanton N, Landry S, Di Bucchianico G, Vallicelli A (Hrsg) Advances in human aspects of transportation. Advances in intelligent systems and computing, Bd. 484. Springer, Cham

2. Arndt S (2011) Evaluierung der Akzeptanz von Fahrassistenzsystemen. Modell zum Kaufverhalten von Endkunden. VS, Wiesbaden

3. Bellem H, Schönenberg T, Krems J, Schrauf M (2016) Objective metrics of comfort: developing a driving style for highly automated vehicles. Transp Res Part F Traffic Psychol Behav 41:45-54. https://doi.org/10.1016/j.trf.2016.05.005

4. Bellem H, Thiel B, Schrauf M, Krems J (2018) Comfort in automated driving: an analysis of preferences for different automated driving styles and their dependence on personality traits. Transp Res Part F Traffic Psychol Behav 55:90-100. https://doi.org/10.1016/j. trf.2018.02.036

5. Bohua S (2018) Analysis of coupling mechanism between driving skill and driving style in driver personalization. In: Conference Paper. 14th International Symposium on Advanced Vehicle Control

6. Clapp JD, Olsen SA, Gayle Beck J, Palyo SA, DeMond MG, Gudmundsdottir B, Marques L (2010) The driving behavior survey: scale construction and validation. J Anxiety Disord 25:96-105

7. Coogan M, Campbell M, Adler T, Forward S (2014) Examining behavioral and attitudinal differences among groups in their traffic safety culture. Transp Res Part F Traffic Psychol Behav 26:303-316

8. Fornell C, Larcker DF (1981) Evaluating structural equation models with unobservable variables and measurement error. J Mark Res 48:39-50

9. Ghazizadeh M, Lee JD, Boyle L (2012) Extending the technology acceptance model to assess automation. Cogn Technol Work 14(1):39-49

10. Glaser W, Waschulewski H (2005) NVENT - Forschungsprojekt Fahrerassistenzsysteme (FAS). Teilprojekt Fahrerverhalten und Mensch-Maschine-Interaktion (FVM) (Arbeitspaket 3200: Validierung und Weiterentwicklung des Bewertungsverfahrens I-TSA (Invent - Traffic Safety Assessment), Abschlussbericht)

11. Groot SD, Ricote F, Winter JCFD (2012) The effect of tire grip on learning driving skill and driving style: a driving simulator study. Transp Res Part F Traffic Psychol Behav 15(4):413-426

12. Haghzare S, Campos J, Bak K, Mihailidis A (2021) Older adults' acceptance of fully automated vehicles: effects of exposure, driving style, age, and driving conditions. Accid Anal Prev. https://doi.org/ 10.1016/j.aap.2020.105919

13. Hair JF, Black WC, Babin BJ, Anderson RE (2010) Multivariate data analysis, 7. Aufl. Prentice Hall, Upper Saddle River
14. Hartwich F, Beggiato M, Krems J (2018) Driving comfort, enjoyment and acceptance of automated driving-Effects of drivers' age and driving style familiarity. Ergonomics 61(8):1017-1032. https:// doi.org/10.1080/00140139.2018.1441448

15. Huysduynen H (2018) Personalized persuasion to increase acceptance of automated driving. Diss, Technische Universiteit Eindhoven

16. Huysduynen H, Terken H, Martens J, Eggen JH (2015) Measuring driving styles: a validation of the multidimensional driving style inventory. In: Automotive UI ' 15 Proceedings of the 7th International Conference on Automotive User Interfaces and Interactive Vehicular Applications https://doi.org/10.1145/2799250.2799266

17. Jian JY, Bisantz AM, Drury CG (1998) Foundations for an empirically determined scale of trust in automated systems. Int J Cogn Ergon 4(1):53-71

18. Karrer K, Glaser C, Clemens C, Bruder C (2009) Technikaffinität erfassen - der Fragebogen TA-EG. Zent Mensch Masch Syst Spektrum 22(29): 196-201

19. Lajunen T, Summala H (1995) Driving experience, personality, and skill and safety-motive dimensions in drivers' self-assessments. Pers Individ Dif 19(3):307-318. https://doi.org/10.1016/ 0191-8869(95)00068-H

20. Lee JD, Liu S, Domeyer J, DinparastDjadid A (2019) Assessing drivers' trust of automated vehicle driving styles with a two-part mixed model of intervention tendency and magnitude. Hum Factors. https://doi.org/10.1177/0018720819880363

21. Lee J, Jang K (2017) A framework for evaluating aggressive driving behaviors based on in-vehicle driving records. Transp Res Part F Traffic Psychol Behav 63:610-619. https://doi.org/10.1016/j.trf. 2017.11.021

22. Manzey D (2008) Systemgestaltung und Automatisierung. In: Badke-Schaub P, Hofinger G, Lauehe K (Hrsg) Human Factors. Psychologie sicheren Handelns in Risikobranchen. Springer, Berlin, Heidelberg, New York

23. Martinussen LM, Møller M, Prato CG (2014) Assessing the relationship between the driver behavior questionnaire and the driver skill inventory: revealing sub-groups of drivers. Transp Res Part F Traffic Psychol Behav 26:82-91

24. Matthews G, Desmond PA, Joyner L, Carcary B, Gilliland K (1996) Validation of the driver stress inventory and driver coping questionnaire. In: International Conference on Traffic and Transport Psychology (ICTTP), Valencia, Spanien

25. Oliveira L, Proctor K, Burns CG, Birrell S (2019) Driving style: how should an automated vehicle behave? Information 10(6):219. https://doi.org/10.3390/info10060219

26. Othersen I (2016) Vom Fahrer zum Denker und Teilzeitlenker. Einflussfaktoren und Gestaltungsmerkmale nutzerorientierter Interaktionskonzepte für die Überwachungsaufgabe des Fahrers im teilautomatisierten Modus. AutoUni Schriftenreihe, Bd. 90. Springer, Wiesbaden

27. Pauzié A (2008) A method to assess the driver mental workload: the Driving Activity Load Index (DALI). IET Intell Transp Syst 2:315-322

28. Rammert W (2003) Technik in Aktion: Verteiltes Handeln in soziotechnischen Konstellationen. In: Christaller T, Wehner J (Hrsg) Autonome Maschinen. Westdeutscher Verlag, Wiesbaden, S 289-315

29. Roidl E, Siebert FW, Höger R (2013) Introducing a multivariate model for predicting driving performance: the role of driving anger and personal characteristics. J Safety Res 47:47-56

30. Roos M, Siegmann M (2020) Technologie-Roadmap für das autonome Autofahren: Eine wettbewerbsorientierte Technik- und Marktstudie für Deutschland. Working Paper Forschungsförderung, Bd. 188. Hans-Böckler-Stiftung, Düsseldorf

31. SAE International (2018) Taxonomy and definitions for terms related to on-road motor vehicle automated driving systems

32. Salzmann F, Doubek F (2020) Modell zur Objektivierung von Fahrstil und Fahrkompetenz. Z Verkehrssicherh 66(3):165-172 
33. Sun X, Li J, Tang P et al (2020) Exploring personalised autonomous vehicles to influence user trust. Cogn Comput 12:1170-1186. https://doi.org/10.1007/s12559-020-09757-x

34. Taubman-Ben Ari O, Skvirsky V (2016) The multidimensional driving style inventory a decade later: review of the literature and reevaluation of the scale. Accid Anal Prev 93:179-188

35. Ulleberg P, Rundmo T (2003) Personality, attitudes and risk perception as predictors of risky driving behaviour among young drivers. Saf Sci 41:427-443

36. Wahlberg A, Dorn L, Kline T (2011) The manchester driver behaviour questionnaire as a predictor of road traffic accidents. Theor Issues Ergon Sci 12(1):66-86
37. Weyer J, Fink R, Adelt F (2014) Human-machine cooperation in smart cars. An empirical investigation of the loss-of-control thesis. Saf Sci 72:199-208

38. Yan F, Liu M, Ding C, Wang Y, Yan L (2019) Driving style recognition based on electroencephalography data from a simulated driving experiment. Front Psychol 10:1254. https://doi.org/10.3389/fpsyg. 2019.01254 